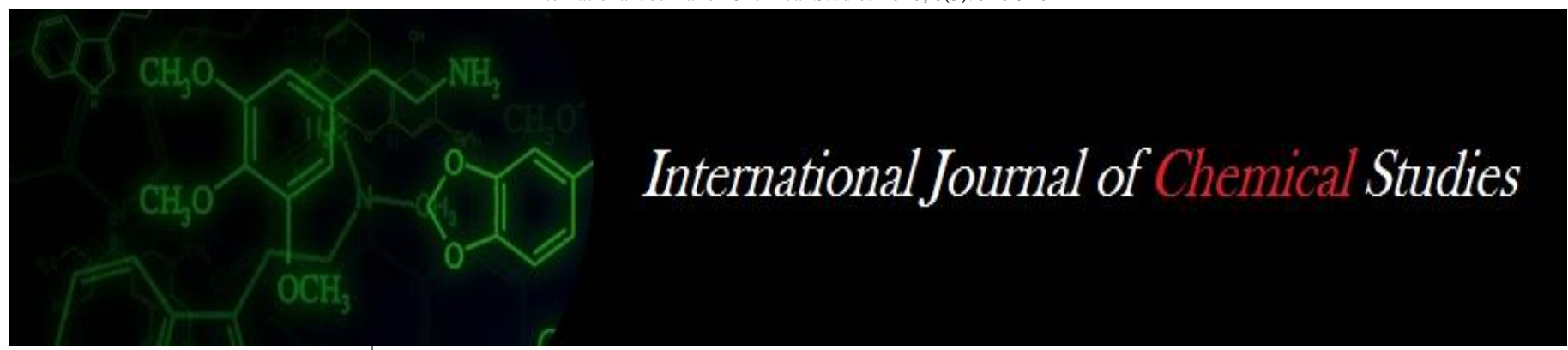

P-ISSN: 2349-8528

E-ISSN: 2321-4902

www.chemijournal.com

IJCS 2020; 8(3): 940-946

(C) 2020 IJCS

Received: 10-03-2020

Accepted: 11-04-2020

Ritesh Kumar Mishra

M. Tech. (Agricultural

Processing and Food

Engineering), IGKVV,

Raipur, Chhattisgarh, India

Dr. S Patel

Head of Department

(Agricultural Processing and

Food Engineering), IGKVV,

Raipur, Chhattisgarh, India

Corresponding Author: Ritesh Kumar Mishra M. Tech. (Agricultural Processing and Food Engineering), IGKVV, Raipur, Chhattisgarh, India

\section{Osmotic dehydration of bottle gourd (Legeneria siceraria)}

\section{Ritesh Kumar Mishra and Dr. S Patel}

DOI: https://doi.org/10.22271/chemi.2020.v8.i31.9320

\begin{abstract}
Osmotic dehydration of fruits and vegetables an effective method for preservation. The Bottle gourd (Legeneria siceraria) belongs to Cucurbitaceous family. Average composition of fresh bottle gourd is constituted by water $(96.0 \%)$, carbohydrates $(2.5 \%)$, fibre $(0.6 \%)$, proteins $(0.2 \%)$ and calcium $(20 \mathrm{mg})$. The bottle gourd is a common vegetable in India subcontinental. It is moderately perishable vegetable. In this report, efforts were made to analyze the effect of different concentration, temperature and mass ratio of fruit to osmotic solution on osmotic dehydration process of bottle gourd cubes. comparative study for different parameters variables (solid gain weight reduction, water loss) was also studies. Five concentration levels of salt solution were $5,10,15,20$, and $25 \%(\mathrm{w} / \mathrm{w})$ used in this study to compare the effect of the concentration of the osmotic agents on osmotic dehydration. Bottle gourd cubes were immersed in the osmotic reagents with a fruit to osmotic solution mass ratio of 10:1, 20:1 and 30:1 soaking experiment were conducted at 40,50 and $60^{\circ} \mathrm{C}$ in a $500 \mathrm{mi}$ capacity water bath. The osmotic dehydration was done for a period of $1,3,5,7$ and $9 \mathrm{~h}$ and replicated thrice. Quality analysis of osmotically dehydrated bottle gourd cubes was done according to ISI (1971) and Ranganna (1991) with 9-point hedonic scale. The moisture content of bottle gourd cubes observed decreases with higher concentration, temperature and mass ratio. The weight reduction, water loss and solid gain were very fast during initial period of osmosis but equilibrium did reach even after $9 \mathrm{~h}$. The solid gain, water loss and weight reduction were observed higher for higher temperature, concentration and mass ratio of bottle gourd cubes. The sensory evaluation performed for the approximately central conditions processing parameters gave average scores of 7 or above.
\end{abstract}

Keywords: Legeneria siceraria, osmotic dehydration, solid gain, water loss, weight reduction

\section{Introduction}

The Bottle gourd (Legeneria Siceraria) belongs to Cucurbitaceous family. The centre of origin has been located as the coastal area of Malabar (North Kerala and humid Forest of Deharadun). It has spread to western countries from India and Africa. The fossil records indicate its culture in India even before 2000BC.

The bottle gourd is a common vegetable in India subcontinental. It is yellowish green, having the shape of bottle. It has white pulp, with white seeds embedded in spongy flesh. They were fairly reach in $\mathrm{Ca}, \mathrm{Fe}$ and $\mathrm{P}$ (Herwig, 2004) ${ }^{[5]}$. The nutritional value of the kernels was equal or better than that of cashew nut and almond kernels. Average composition of fresh bottle gourd (depending on variety, climate and ripeness) is constituted by water (96.0\%), carbohydrates $(2.5 \%)$, fibre $(0.6 \%)$, proteins $(0.2 \%)$ and calcium $(20 \mathrm{mg})$ (Herwig, 2004) ${ }^{[5]}$. Bottle gourd mostly cultivated Uttar Pradesh, Gujarat, Assom, Meghalaya and Rajsthan.

The goal of food preservation is to increase the time for keeping food safe while retaining quality and nutrients. Fruits and vegetables are highly perishable due to their high moisture content. Decreasing the moisture content of fresh food to make them less perishable is a simple way to preserve these foods. The removal of moisture presents the moisture-mediated deteriorative reactions. It brings about substantial reduction in weight and volume, minimizing packing, storage and transportation costs and enables storability of the product under ambient temperatures, Dehydration increases the storage ability of fruits and vegetables making them available throughout the year. Dehydrated products also play a great role in processed foods of all kinds (i.e., in soups) and the way to achieve high quality dehydrated products are desired. Practically any fruit and vegetable can be processed, but some important factors which determine whether it is worthwhile are: 
a. The demand for a particular fruit or vegetable in the processed form;

b. The quality of the raw material, i.e. whether it can withstand processing;

c. Regular supplies of the raw material.

Osmotic dehydration is a useful technique for the concentration of fruit and vegetables, released by placing the solid food, whole or in pieces, in sugars or salts aqueous solution of high osmotic pressure, it gives rise to at least two major simultaneous counter-current flows: a significant water flow out of the food into the solution and a transfer of solute from the solution into the food. The effects of osmotic dehydration as pre-treatment are mainly related to the improvement of some nutritional, organoleptic and functional properties of the product. As osmotic dehydration is effective at ambient temperature, heat damage to colour and flavor is minimized and the high concentration of sugar surrounding fruit and vegetables pieces prevents discoloration.

Keeping this in the mind the present study has been conducted with the following objectives:

1. To study the osmotic dehydration characteristics of bottle gourd cubes.

2. To study the quality of osmotically dehydrated bottle gourd cubes.

\section{Materials and methods}

The work was carried out in the Department of Agricultural Processing and Food Engineering, Indira Gandhi Agricultural University, Raipur (C.G.).

\subsection{Preparation of the samples and osmotic solution}

Bottle gourd fruits were washed well in running water to remove the surface dirt etc. Washed fruits were taken out from the water and spread under fan on a paper towel and blotted to remove the surface water. The fruits were then peeled off with the help of a stainless-steel hand peeler and both ends were trimmed off using a stainless-steel knife. Pieces of bottle gourd cubes $(1 \mathrm{cmx} 1 \mathrm{cmx} 1 \mathrm{~cm})$ were then cut out using a ruler and knife. The cubes so obtained were used immediately for the experimentation.

Common salt ( $98 \%$ of minimum purity in sodium chloride) was used as osmotic agent salt solution of different concentration viz. 5\%, 10\%, 15\%, 20\% and 25\%(w/w) were prepared using distilled water at room temperature (Sereno et.al., 2004) ${ }^{[12]}$.

\subsection{Experimental set up for preliminary osmotic dehydration experiments}

The experimental set up consisted of glass beakers of $500 \mathrm{ml}$ capacity, a water bath of heating capacity $110^{\circ} \mathrm{C}$ having temperature control arrangement $\left( \pm 2^{\circ} \mathrm{C}\right)$ and mercury in glass thermometer $\left(10\right.$ to $\left.200^{\circ} \mathrm{C}\right)$.

\subsection{Experimental Procedure}

Osmotic dehydration was carried out with commercially available table salt (98\% of minimum purity in sodium chloride) as osmotic agent. Five concentration levels of salt solution were used in this study to compare the effect of concentration of osmotic agent on osmotic dehydration. The salt solution with $5,10,15,20$, and $25 \%$ (w/w) concentration were prepared and bottle gourd cubes were immersed in the osmotic reagents with a fruit to osmotic solution mass ratio of 10;1, 20:1 and 30:1. The temperature of osmotic solution were 40,50 and $60^{\circ} \mathrm{C}$. The osmotic dehydration done for a period of 1, 3, 5, 7 and $9 \mathrm{~h}$ and repeated thrice. Afterwards samples were rinsed and wiped with tissues.

Different concentrations of osmotic solutions were prepared and the solution was mixed with bottle gourd cubes in selected mass ratios. The beaker was placed in hot water-bath maintained at desired temperature for soaking. After every 1, 3, 5, 7 and $9 \mathrm{~h}$ sample was taken out and moisture content was determined by standard method (Ranganna 1995) ${ }^{[10]}$. Water loss $(\%)$, solid gain $(\%)$, weight reduction $(\%)$ for each sample was calculated.

\subsection{Variables and their ranges}

In the osmosis process various factors are involved which directly or indirectly affect the osmosis action. Based on the preliminary experiments the following independent and dependent variables are selected in the present investigation. The independent variables are type of osmotic solution salt, initial moisture content, soaking temperature and soaking time, mass ratio and concentration of osmotic agent.

- Type of Osmotic solution: Common salt (minimum 98\% of purity)

- Concentration of Osmotic agent: 5, 10, 15, 20, 25\%(w/w)

- Time of osmotic dehydration: 1, 3, 5, 7 and $9 \mathrm{~h}$

- Mass ratio (sample to osmotic solution): 1:10, 1:20 and $1: 30$.

- Temperature of osmotic solution: 40,50 and $60^{\circ} \mathrm{C}$.

The dependent variables studies were,

a) Water loss

b) Weight reduction

c) Solid gain and

d) Moisture content after dehydration

\subsection{Measurement of dependent variables} 2.5.1 Moisture content determination

Test sample of $100 \mathrm{~g}$ was kept for $24 \mathrm{~h}$ in a hot air electric oven maintained at $105^{\circ} \mathrm{C}$. After $24 \mathrm{~h}$, sample was drawn from the oven and placed in a desiccator for cooling. After cooling the weight of the sample was taken precisely. The loss was determined and moisture content was calculated using the following expression:

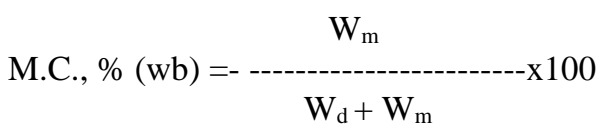

Where,

M.C. = Moisture content, $\%$

$\mathrm{W}_{\mathrm{m}}=$ Weight of moisture,

$\mathrm{W}_{\mathrm{d}}=$ Weight of dry matter.

\subsubsection{Determination of solid gain}

The solid gain bottle gourd cubes after osmotic dehydration were calculated using following equation (Nieto et al., 2004) [7].

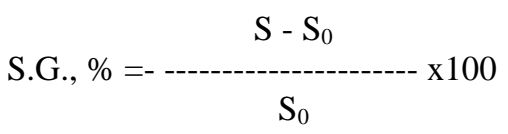

Where,

S.G. = Solid gain $(\%)$,

$\mathrm{S}=$ mass solids after osmosis $(\mathrm{g})$,

$\mathrm{S}_{0}=$ mas solids before osmosis $(\mathrm{g})$. 


\subsubsection{Determination of weight reduction}

The weight reduction of bottle gourd cubes after osmotic dehydration were calculated using following equation (Nieto et al., 2004) ${ }^{[7]}$.

$$
\text { W.R., \% =- -------------------- X } 100
$$

Where,

W.R. $=$ Weight reduction $(\%)$,

$\mathrm{S}=$ initial weight of the sample $(\mathrm{g})$,

$\mathrm{S}_{0}=$ weight of the sample at time $\mathrm{t}(\mathrm{g})$.

\subsubsection{Determination of water loss}

The water loss of bottle gourd cubes after osmotic dehydration were calculated using following equation (Nieto et al., 2004) ${ }^{[7]}$.

$$
\text { W.L., }(\%)=-\mathrm{SG}-\mathrm{WR}
$$

\subsection{Quality analysis}

Quality analysis of osmotically dehydrated bottle gourd cubes was done according to ISI (1971) and Ranganna (1991) with 9-point hedonic scale. The average sensory scores for different attributes, viz., appearance, colour, texture and overall acceptability for the bottle gourd cubes of size $1 \mathrm{~cm}$ were given. Quality analysis was performed by the panel of semi-trained judges drawn from the employees and students of Faculty of Agricultural Engineering, Indira Gandhi Agricultural University, Raipur (C.G.). The panelists were provided the product evaluation sheet

\section{Results and Discussion \\ 3.1 Osmotic dehydration experiment}

Bottle gourd cubes of $1 \mathrm{~cm}$ size were subjected to osmatic dehydration using commercial salt for osmatic solution of different concentrations. The ranges were temperature 40, 50 and $60^{\circ} \mathrm{C}$ osmotic dehydration time $1,3,5,7$ and $9 \mathrm{~h}$, mass ratio of osmatic solution to the bottle gourd cubes (fruit cube): $30: 1,20: 1$ and 10:1 and concentration of osmotic solution 5, $10,15,20$ and $25 \%$.

\subsubsection{Moisture studies}

The moisture content of bottle gourd cubes was calculated during osmotic dehydration experiment each after 1, 3, 5, 7 and $9 \mathrm{~h} \mathrm{r}$ respectively. The values of moisture content are presented in Table 3.1 through 3.3.

The effect of dehydration time on loss of moisture indicates that the as the time increases the loss of moisture increases. This was true for all the combination of mass ratio and temperature set. The values of moisture content at $25 \%$ concentration of osmotic solution were $68.3 \%, 67.7 \%, 68.9 \%$ after $1 \mathrm{~h}$ dehydration; $54.9 \%, 60.9 \%, 60.9 \%(w b)$ after $3 \mathrm{~h}$; $53.8 \%, 56.4 \%$ and $55.2 \%(\mathrm{wb})$ after $5 \mathrm{~h} ; 51.7 \%, 50.8 \%$ and $53.2 \%(w b)$ after $7 \mathrm{~h} ; 50.1 \%, 49.9 \%$ and $46.9 \%$ (wb) after $9 \mathrm{~h}$ of dehydration for the mass ratio of 10:1, 20:1 and 30:1 respectively for the osmotic temperature of $60^{\circ} \mathrm{C}$. The highest removal of moisture was in the initial stage of dehydration i.e., upto $1 \mathrm{~h}$ of dehydration as compared to final stage of dehydration i.e., from 3 to $9 \mathrm{~h}$ in the case of $60^{\circ} \mathrm{C}$ and similar trends were followed for $50^{\circ} \mathrm{C}$ and $40^{\circ} \mathrm{C}$. Similarly, while comparing different osmotic temperatures for the same set of mass ratio (30:1) the value of moisture content at $25 \%$ concentration of osmotic solution were $46.9 \%$ (wb) $60^{\circ} \mathrm{C}$; $49.3 \%$ at $50^{\circ} \mathrm{C} ; 51.7 \%(\mathrm{wb})$ at $40^{\circ} \mathrm{C}$ which were dehydrated for $9 \mathrm{~h}$. this suggested that at same concentration of osmotic solution, higher temperature is responsible for higher removal of moisture from the sample.

\begin{tabular}{|c|c|c|c|c|c|c|c|c|c|c|c|c|c|c|c|c|c|c|c|c|}
\hline \multirow{4}{*}{$\mathbf{T}$} & \multicolumn{20}{|c|}{ Mass ratio } \\
\hline & & & 10:1 & & & & & $20: 1$ & & & \multirow{2}{*}{\multicolumn{5}{|c|}{$\frac{\text { Concentration (\%) }}{\text { Concen }}$}} & \multirow{2}{*}{\multicolumn{5}{|c|}{$\frac{\text { Mean }}{\text { Concentration }(\%)}$}} \\
\hline & \multicolumn{5}{|c|}{ Concentration (\%) } & \multicolumn{5}{|c|}{ Concentration (\%) } & & & & & & & & & & \\
\hline & 5 & 10 & 15 & 20 & 25 & 5 & 10 & 15 & 20 & 25 & 5 & 10 & 15 & 20 & 25 & 5 & 10 & 15 & 20 & 25 \\
\hline 0 & 96.3 & 96.3 & 96.3 & 96.3 & 96.3 & 96.3 & 96.3 & 96.3 & 96.3 & 96.3 & 96.3 & 96.3 & 96.3 & 96.3 & 96.3 & 96.3 & 96.3 & 96.3 & 96.3 & 96.3 \\
\hline 1 & 93.4 & 91.6 & 85.4 & 80.1 & 75.2 & 93.4 & 90.2 & 85.7 & 80.2 & 74.8 & 93.0 & 84.1 & 82.3 & 73.4 & 67.4 & 93.3 & 88.6 & 84.5 & 77.9 & 72.5 \\
\hline 3 & 92.2 & 88.3 & 82.8 & 71.3 & 62.8 & 91.4 & 85.9 & 82.4 & 71.9 & 62.8 & 91.9 & 82.8 & 89.2 & 72.1 & 62.6 & 91.8 & 85.7 & 84.8 & 71.8 & 62.7 \\
\hline 5 & 91.6 & 85.3 & 79.1 & 75.3 & 56.4 & 90.8 & 84.2 & 77.5 & 75.0 & 58.2 & 91.4 & 82.5 & 78.9 & 67.2 & 57.3 & 91.3 & 84.0 & 78.5 & 72.5 & 57.3 \\
\hline 7 & 90.6 & 82.3 & 75.2 & 59.6 & 53.2 & 90.1 & 82.2 & 73.8 & 65.1 & 51.7 & 90.8 & 82.2 & 78.1 & 64.8 & 52.6 & 90.5 & 82.2 & 75.7 & 63.2 & 52.5 \\
\hline 9 & 89.6 & 80.7 & 73.2 & 56.2 & 53.7 & 88.8 & 80.1 & 72.9 & 62.5 & 52.8 & 84.6 & 80.3 & 77.8 & 65.2 & 51.7 & 87.7 & 80.4 & 74.6 & 61.3 & 52.7 \\
\hline $\bar{M}$ & 92.3 & 87.4 & 82.0 & 73.1 & 66.3 & 91.8 & 86.5 & 81.4 & 75.2 & 66.1 & 91.3 & 84.7 & 83.8 & 73.2 & 64.7 & & & & & \\
\hline
\end{tabular}

Table 3.1: Mean values of moisture content $(\%, w b)$ of osmotically dehydrated bottle gourd at 40 Degree Celsius

\begin{tabular}{|c|c|c|c|c|c|c|c|c|c|c|c|c|c|c|c|c|c|c|c|c|}
\hline \multirow{4}{*}{$\mathbf{T}$} & \multicolumn{20}{|c|}{ Mass ratio } \\
\hline & & & 10:1 & & & & & 20:1 & & & & & 30:1 & & & & & Mean & & \\
\hline & \multicolumn{5}{|c|}{ Concentration (\%) } & \multicolumn{5}{|c|}{ Concentration (\%) } & \multicolumn{5}{|c|}{ Concentration (\%) } & \multicolumn{5}{|c|}{ Concentration (\%) } \\
\hline & 5 & 10 & 15 & 20 & 25 & 5 & 10 & 15 & 20 & 25 & 5 & 10 & 15 & 20 & 25 & 5 & 10 & 15 & 20 & 25 \\
\hline 0 & 96.0 & 96.0 & 96.0 & 96.0 & 96.0 & 96.0 & 96.0 & 96.0 & 96.0 & 96.0 & 96.0 & 96.0 & 96.0 & 96.0 & 96.0 & 96.0 & 96.0 & 96.0 & 96.0 & 96.0 \\
\hline 1 & 92.2 & 88.8 & 85.1 & 80.5 & 75.6 & 92.6 & 88.1 & 85.3 & 79.2 & 75.5 & 92.1 & 83.7 & 81.6 & 73.8 & 65.9 & 92.3 & 86.9 & 84.0 & 77.8 & 72.3 \\
\hline 3 & 90.6 & 86.6 & 81.8 & 72.2 & 62.1 & 91.6 & 86.3 & 81.9 & 72.0 & 61.8 & 91.3 & 81.2 & 78.4 & 71.2 & 60.5 & 91.2 & 84.7 & 80.7 & 71.8 & 61.5 \\
\hline 5 & 90.6 & 84.5 & 77.3 & 66.3 & 58.3 & 90.3 & 84.3 & 78.2 & 63.3 & 67.4 & 92.3 & 81.5 & 75.2 & 78.2 & 65.6 & 91.1 & 83.4 & 76.9 & 69.3 & 63.8 \\
\hline 7 & 89.6 & 82.2 & 74.3 & 59.2 & 51.9 & 88.6 & 82.4 & 73.8 & 59.1 & 51.7 & 88.0 & 81.7 & 72.8 & 65.1 & 51.2 & 88.7 & 82.1 & 73.6 & 61.1 & 51.6 \\
\hline 9 & 88.6 & 80.5 & 72.1 & 55.8 & 52.3 & 89.3 & 80.4 & 73.1 & 56.5 & 51.2 & 84.3 & 80.2 & 72.3 & 60.6 & 49.3 & 87.4 & 80.4 & 72.5 & 57.6 & 50.9 \\
\hline M & 91.3 & 86.4 & 81.1 & 71.7 & 66.0 & 91.4 & 86.3 & 81.4 & 71.0 & 67.3 & 90.7 & 84.1 & 79.4 & 74.2 & 64.8 & & & & & \\
\hline
\end{tabular}

Table 3.2: Mean values of moisture content $(\%, w b)$ of osmotically dehydrated bottle gourd at 50 Degree Celsius 
Table 3.3: Mean values of moisture content $(\%, w b)$ of osmotically dehydrated bottle gourd at 60 Degree Celsius

\begin{tabular}{|c|c|c|c|c|c|c|c|c|c|c|c|c|c|c|c|c|c|c|c|c|}
\hline \multirow{4}{*}{$\mathbf{T}$} & \multicolumn{20}{|c|}{ Mass ratio } \\
\hline & \multicolumn{5}{|c|}{ 10:1 } & \multirow{2}{*}{\multicolumn{5}{|c|}{$\begin{array}{c}\text { 20:1 } \\
\text { Concentration }(\%)\end{array}$}} & \multicolumn{5}{|c|}{ 30:1 } & \multicolumn{5}{|c|}{ Mean } \\
\hline & \multicolumn{5}{|c|}{ Concentration (\%) } & \multicolumn{4}{|c|}{ Concentration (\%) } & & \multicolumn{5}{|c|}{ Concentration (\%) } & \multicolumn{5}{|c|}{ Concentration (\%) } \\
\hline & 5 & 10 & 15 & 20 & 25 & 5 & 10 & 15 & 20 & 25 & 5 & 10 & 15 & 20 & 25 & 5 & 10 & 15 & 20 & 25 \\
\hline 0 & 95.6 & 95.6 & 95.6 & 95.6 & 95.6 & 95.6 & 95.6 & 95.6 & 95.6 & 95.6 & 95.6 & 95.6 & 95.6 & 95.6 & 95.6 & 95.6 & 95.6 & 95.6 & 95.6 & 95.6 \\
\hline 1 & 92.2 & 88.5 & 85.6 & 82.1 & 68.3 & 91.3 & 87.5 & 84.8 & 76.8 & 67.7 & 89.0 & 82.3 & 70.8 & 70.4 & 68.9 & 90.8 & 86.1 & 80.4 & 76.4 & 68.3 \\
\hline 3 & 91.4 & 87.3 & 81.9 & 71.2 & 54.9 & 90.1 & 86.4 & 81.2 & 71.9 & 60.9 & 89.2 & 80.0 & 76.3 & 69.0 & 60.9 & 90.2 & 84.6 & 79.8 & 70.7 & 58.9 \\
\hline 5 & 89.3 & 84.2 & 78.3 & 66.3 & 53.8 & 88.6 & 84.2 & 76.6 & 74.4 & 56.4 & 88.5 & 80.4 & 74.4 & 63.7 & 55.2 & 88.8 & 82.9 & 76.4 & 68.1 & 55.1 \\
\hline 7 & 87.5 & 82.2 & 73.9 & 56.8 & 51.7 & 86.5 & 81.9 & 74.2 & 59.1 & 50.8 & 88.1 & 80.2 & 72.3 & 59.3 & 53.2 & 87.4 & 81.4 & 73.5 & 58.4 & 51.9 \\
\hline 9 & 89.1 & 81.3 & 71.9 & 54.6 & 50.1 & 86.8 & 79.8 & 72.2 & 55.0 & 49.9 & 82.1 & 78.2 & 69.1 & 51.4 & 46.9 & 86.0 & 79.8 & 71.1 & 53.7 & 49.0 \\
\hline $\bar{M}$ & 90.9 & 86.5 & 81.2 & 71.1 & 62.4 & 89.8 & 85.9 & 80.8 & 72.1 & 63.6 & 88.8 & 82.8 & 76.4 & 68.2 & 63.5 & & & & & \\
\hline
\end{tabular}

\subsubsection{Solid gain}

The solid gain of bottle gourd cubes was calculated during osmotic dehydration experiment for each after $1,3,5,7$, and 9 $\mathrm{h}$ respectively. The mean values of solid gain for the different set of experiments are presented in Table 3.4 through 3.6.

Table 4.4: Mean values of solid gain (\%) of osmotically dehydrated bottle gourd at 40 Degree Celcious

\begin{tabular}{|c|c|c|c|c|c|c|c|c|c|c|c|c|c|c|c|c|c|c|c|c|}
\hline \multirow{4}{*}{$\mathbf{T}$} & \multicolumn{20}{|c|}{ Mass ratio } \\
\hline & \multicolumn{5}{|c|}{ 10:1 } & \multicolumn{5}{|c|}{$20: 1$} & \multicolumn{5}{|c|}{ 30:1 } & \multicolumn{5}{|c|}{ Mean } \\
\hline & \multicolumn{5}{|c|}{ Concentration (\%) } & \multicolumn{5}{|c|}{ Concentration $(\%)$} & \multicolumn{5}{|c|}{ Concentration (\%) } & \multicolumn{5}{|c|}{ Concentration $(\%)$} \\
\hline & 5 & 10 & 15 & 20 & 25 & 5 & 10 & 15 & 20 & 25 & 5 & 10 & 15 & 20 & 25 & 5 & 10 & 15 & 20 & 25 \\
\hline 1 & 4.7 & 5.0 & 5.8 & 6.5 & 8.2 & 4.7 & 5.8 & 6.1 & 6.4 & 8.1 & 6.4 & 9.8 & 7.0 & 7.0 & 9.1 & 5.2 & 6.8 & 6.3 & 6.6 & 8.4 \\
\hline 3 & 5.1 & 6.0 & 5.9 & 7.2 & 8.8 & 4.7 & \begin{tabular}{|l|}
5.9 \\
\end{tabular} & 6.2 & 7.2 & 9.2 & 6.6 & 7.9 & 8.1 & 9.1 & 10.1 & 5.5 & 6.6 & 6.7 & 7.8 & 9.4 \\
\hline 5 & 5.0 & 6.0 & 8.0 & 10.0 & 11.6 & 5.0 & 5.9 & 7.8 & 9.5 & 11.4 & 7.3 & 8.1 & 11.6 & 12.7 & 14.4 & 5.8 & 6.7 & 9.1 & 10.7 & 12.5 \\
\hline 7 & 5.3 & 6.0 & 9.4 & 12.6 & 14.0 & 5.7 & 6.1 & 9.9 & 12.1 & 13.9 & 8.3 & 9.0 & 14.1 & 12.8 & 18.5 & 6.4 & $\begin{array}{l}7.0 \\
\end{array}$ & 11.1 & 12.5 & 15.5 \\
\hline 9 & 5.9 & 8.1 & 11.7 & 12.7 & 19.5 & 6.5 & 8.2 & 11.7 & 16.9 & 19.6 & 10.3 & 12.3 & 15.6 & 17.8 & 19.8 & 7.6 & \begin{tabular}{|l|}
9.5 \\
\end{tabular} & 13.0 & 15.8 & 19.6 \\
\hline $\mathrm{M}$ & 5.2 & 6.2 & 8.2 & 9.8 & 12.4 & 5.3 & 6.4 & 8.3 & 10.4 & 12.4 & 7.8 & 9.4 & 11.3 & 11.9 & 14.4 & & & & & \\
\hline
\end{tabular}

Table 4.5: Mean values of solid gain (\%) of osmotically dehydrated bottle gourd at 50 Degree Celcious

\begin{tabular}{|c|c|c|c|c|c|c|c|c|c|c|c|c|c|c|c|c|c|c|c|c|}
\hline \multirow{4}{*}{$\mathbf{T}$} & \multicolumn{20}{|c|}{ Mass ratio } \\
\hline & \multicolumn{5}{|c|}{$10: 1$} & \multicolumn{5}{|c|}{$\begin{array}{l}20: 1 \\
\end{array}$} & \multicolumn{5}{|c|}{$\begin{array}{c}30: 1 \\
\end{array}$} & \multicolumn{5}{|c|}{ Mean } \\
\hline & \multicolumn{5}{|c|}{ Concentration (\%) } & \multicolumn{5}{|c|}{ Concentration (\%) } & \multicolumn{5}{|c|}{ Concentration (\%) } & \multicolumn{5}{|c|}{ Concentration (\%) } \\
\hline & 5 & 10 & 15 & 20 & 25 & 5 & 10 & 15 & 20 & 25 & 5 & 10 & 15 & 20 & 25 & 5 & 10 & 15 & 20 & 25 \\
\hline 1 & 5.3 & 5.8 & 6.4 & 6.8 & 8.6 & 5.7 & 6.3 & 6.7 & 6.9 & 8.8 & 6.4 & 8.3 & 7.0 & 7.0 & 9.2 & 5.8 & 6.8 & 6.7 & 6.9 & 8.8 \\
\hline 3 & 5.7 & 6.3 & 6.9 & 8.2 & 9.4 & 5.8 & 6.6 & 7.1 & 8.2 & 10.2 & 6.7 & 8.0 & 8.4 & 9.1 & 10.7 & 6.1 & 7.0 & 7.4 & 8.5 & 10.1 \\
\hline 5 & 6.2 & 7.0 & 8.3 & 10.1 & 12.9 & 6.7 & 7.5 & 10.0 & 11.2 & 12.8 & 7.8 & 8.6 & 12.0 & 13.1 & 15.0 & 6.9 & 7.7 & 10.1 & 11.5 & 13.6 \\
\hline 7 & 6.7 & 7.3 & 10.5 & 12.6 & 15.8 & 7.9 & 8.5 & 12.1 & 14.4 & 16.2 & 9.4 & 10.4 & 14.5 & 12.9 & 18.9 & 8.0 & 8.7 & 12.4 & 13.3 & 16.9 \\
\hline 9 & 8.1 & 9.9 & 12.0 & 13.3 & 20.3 & 8.6 & 10.3 & 13.7 & 17.5 & 20.4 & 11.1 & 13.3 & 16.1 & 18.1 & 20.6 & 9.2 & $\begin{array}{ll}11.1 \\
\end{array}$ & 13.9 & 16.3 & 20.4 \\
\hline $\bar{M}$ & 6.4 & 7.3 & 8.8 & 10.2 & 13.4 & 6.9 & 7.8 & 9.9 & 11.6 & 13.7 & 8.3 & 9.7 & 11.6 & 12.0 & 14.9 & & & & & \\
\hline
\end{tabular}

Table 4.6: Mean values of solid gain (\%) of osmotically dehydrated bottle gourd at 60 Degree Celcious

\begin{tabular}{|c|c|c|c|c|c|c|c|c|c|c|c|c|c|c|c|c|c|c|c|c|}
\hline \multirow{4}{*}{$\mathbf{T}$} & \multicolumn{20}{|c|}{ Mass ratio } \\
\hline & \multicolumn{5}{|c|}{ 10:1 } & \multicolumn{5}{|c|}{$20: 1$} & \multirow{2}{*}{\multicolumn{5}{|c|}{$\begin{array}{l}30: 1 \\
\end{array}$}} & \multicolumn{5}{|c|}{ Mean } \\
\hline & \multicolumn{5}{|c|}{ Concentration (\%) } & \multicolumn{5}{|c|}{ Concentration (\%) } & \multicolumn{2}{|c|}{ Concentration (\%) } & & & & \multicolumn{5}{|c|}{ Concentration (\%) } \\
\hline & 5 & 10 & 15 & 20 & 25 & 5 & 10 & 15 & 20 & 25 & 5 & 10 & 15 & 20 & 25 & 5 & 10 & 15 & 20 & 25 \\
\hline 1 & 5.9 & 6.6 & 7.1 & 7.2 & 9.0 & 6.7 & 6.8 & 7.3 & 7.5 & 9.5 & 6.5 & 6.8 & 7.1 & 7.1 & 9.3 & 6.4 & 6.7 & 7.1 & 7.2 & 9.3 \\
\hline 3 & 6.3 & 6.7 & 7.8 & 9.2 & 10.0 & 6.8 & 7.4 & 8.1 & 9.2 & 11.2 & 6.9 & 8.0 & 8.6 & 9.1 & 11.3 & 6.7 & 7.4 & 8.2 & 9.2 & 10.8 \\
\hline 5 & 7.4 & 8.0 & 8.6 & 10.3 & 14.1 & 8.4 & 9.0 & 12.2 & 12.9 & 14.2 & 8.2 & 9.2 & 12.4 & 13.4 & 15.6 & 8.0 & 8.7 & 11.1 & 12.2 & 14.6 \\
\hline 7 & 8.1 & 8.7 & 10.8 & 12.6 & 17.5 & 10.1 & 10.9 & 14.3 & 16.7 & 18.5 & 10.6 & 11.7 & 14.9 & 13.3 & 19.2 & 9.6 & 10.4 & 13.3 & 14.2 & 18.4 \\
\hline 9 & 10.3 & 11.6 & 12.2 & 14.8 & 20.6 & 10.6 & 12.5 & 15.6 & 17.9 & 20.8 & 11.8 & 14.2 & 16.6 & 18.3 & 21.4 & 10.9 & 12.8 & 14.8 & 17.0 & 20.9 \\
\hline $\bar{M}$ & 7.6 & 8.3 & 9.3 & 10.8 & 14.2 & 8.5 & 9.3 & 11.5 & 12.8 & 14.8 & 8.8 & 10.0 & 11.9 & 12.2 & 15.4 & & & & & \\
\hline
\end{tabular}

The effect of dehydration time on solid gain indicates that as the time increases the solid gain increases. This was true for all the combination of mass ratio and temperature set. While comparing different osmotic temperatures for the same set of mass ratio (30:1) the value of solid gain at $25 \%$ concentration of osmotic solution were $21.4 \%(\mathrm{wb})$ at $60^{\circ} \mathrm{C}, 20.6 \%(\mathrm{wb})$ at $50^{\circ} \mathrm{C}, 19.8 \%(\mathrm{wb})$ at $40^{\circ} \mathrm{C}$ which were dehydrated for $9 \mathrm{~h}$. This suggests that at same concentration of osmotic solution, higher temperature is responsible for increasing solid gain. The same trends were obtained three mass ratio 10:1, 20:1 and 30:1. As can be seen the values of solid gain at $25 \%$ concentration of osmotic solution were $20.6 \%, 20.8 \%$ and
$21.4 \%$ at $60^{\circ} \mathrm{C}, 20.3 \%, 20.4 \%$ and $20.6 \%$ at $50^{\circ} \mathrm{C}, 19.5 \%$, $19.6 \%$ and $19.8 \%$ at $40^{\circ} \mathrm{C}$ for the mass ratio of $10: 1,20: 1$ and 30:1 respectively which were dehydrated for $9 \mathrm{~h}$. the solid gain for samples dehydrated at 30:1 mass ratio were more to samples dehydrated at 10:1 mass ratio comparatively, keeping other variables constant. It might be due to higher mobility of viscous liquid.

\subsubsection{Water loss}

The kinetic of water loss at different process conditions was obtained. The mean value of water loss for the different set of experiments for presented in Table 3.7 through 3.9. 
It can be depicted that the increase in the concentration of osmotic solution the loss of water is the removal of water increased. The maximum water loss was $59.9 \%$ found for samples osmotically dehydrated at $60^{\circ} \mathrm{C}$ for $9 \mathrm{~h}$ at $30: 1$ fruit to osmotic solution of $25 \%$ concentration. The minimum $14.60 \%$ water loss was found for samples osmotically dehydrated at $40^{\circ} \mathrm{C}$ for $1 \mathrm{~h}$ at $10: 1$ fruit to osmotic solution of $5 \%$ concentration.

Table 3.7: Mean values of water loss (\%) of osmotically dehydrated bottle gourd at 40 Degree Celsius

\begin{tabular}{|c|c|c|c|c|c|c|c|c|c|c|c|c|c|c|c|c|c|c|c|c|}
\hline \multirow{4}{*}{$\mathbf{T}$} & \multicolumn{20}{|c|}{ Mass ratio } \\
\hline & \multicolumn{5}{|c|}{ 10:1 } & \multicolumn{5}{|c|}{$20: 1$} & \multicolumn{5}{|c|}{ 30:1 } & \multicolumn{5}{|c|}{ Mean } \\
\hline & \multicolumn{5}{|c|}{ Concentration (\%) } & \multicolumn{5}{|c|}{ Concentration (\%) } & \multicolumn{5}{|c|}{ Concentration (\%) } & \multicolumn{5}{|c|}{ Concentration (\%) } \\
\hline & 5 & 10 & 15 & 20 & 25 & 5 & 10 & 15 & 20 & 25 & 5 & 10 & 15 & 20 & 25 & 5 & 10 & 15 & 20 & 25 \\
\hline 1 & 14.6 & 15.9 & 17.8 & 18.7 & 23.4 & 17.6 & 20.6 & 21.4 & 22.7 & 25.8 & 19.5 & 22.2 & 23.8 & 23.6 & 27.5 & 17.2 & 19.6 & 21.0 & 21.7 & 25.6 \\
\hline 3 & 16.9 & 18.5 & 20.1 & 22.9 & 25.5 & 18.4 & 22.3 & 24.1 & 28.2 & 31.0 & 22.3 & 25.7 & 27.5 & 30.6 & 32.8 & 19.2 & 22.2 & 23.9 & 27.2 & 29.8 \\
\hline 5 & 18.2 & 20.4 & 23.8 & 27.5 & 31.3 & 20.2 & 25.3 & 31.1 & 34.3 & 36.6 & 25.8 & 29.3 & 34.6 & 38.4 & 43.5 & 21.4 & 25.0 & 29.8 & 33.4 & 37.1 \\
\hline 7 & 17.4 & 19.2 & 22.2 & 24.9 & 28.2 & 19.5 & 23.8 & 27.0 & 30.9 & 34.0 & 23.3 & 26.5 & 30.2 & 33.1 & 38.8 & 20.1 & 23.2 & 26.5 & 29.6 & 33.7 \\
\hline 9 & 19.9 & 23.2 & 27.3 & 31.4 & 37.6 & 22.5 & 28.4 & 32.8 & 40.3 & 43.5 & 27.6 & 31.5 & 36.4 & 41.2 & 44.9 & 23.3 & 27.7 & 32.2 & 37.6 & 42.0 \\
\hline M & \begin{tabular}{|l|l}
17.4 \\
\end{tabular} & \begin{tabular}{|l|l}
19.4 \\
\end{tabular} & 22.2 & 25.1 & 29.2 & 19.6 & 24.1 & 27.3 & 31.3 & 34.2 & 23.7 & 27.0 & 30.5 & 33.4 & 37.5 & & & & & \\
\hline
\end{tabular}

Table 3.8: Mean values of water loss (\%) of osmotically dehydrated bottle gourd at 50 Degree Celsius

\begin{tabular}{|c|c|c|c|c|c|c|c|c|c|c|c|c|c|c|c|c|c|c|c|c|}
\hline \multirow{4}{*}{$\mathbf{T}$} & \multicolumn{20}{|c|}{ Mass ratio } \\
\hline & \multicolumn{5}{|c|}{$10: 1$} & \multirow{2}{*}{\multicolumn{5}{|c|}{\begin{tabular}{|c|}
$20: 1$ \\
Concentration $(\%)$ \\
\end{tabular}}} & \multirow{2}{*}{\multicolumn{5}{|c|}{$\begin{array}{c}\text { 30:1 } \\
\text { Concentration (\%) }\end{array}$}} & \multirow{2}{*}{\multicolumn{5}{|c|}{$\begin{array}{c}\text { Mean } \\
\text { Concentration (\%) }\end{array}$}} \\
\hline & & Conce & ntrati & 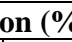 & & & & & & & & & & & & & & & & \\
\hline & 5 & 10 & 15 & 20 & 25 & 5 & 10 & 15 & 20 & 25 & 5 & 10 & 15 & 20 & 25 & 5 & 10 & \begin{tabular}{|c|}
15 \\
\end{tabular} & 20 & 25 \\
\hline 1 & 15.6 & 19.6 & 23.8 & 26.5 & 29.1 & 18.3 & 21.2 & 25.1 & 25.9 & 30.7 & 20.9 & 24.1 & 26.2 & 26.8 & \begin{tabular}{|l|}
33.1 \\
\end{tabular} & 18.3 & 21.6 & 25.0 & 26.4 & 31.0 \\
\hline 3 & 20.5 & 22.7 & 24.2 & 28.9 & 31.4 & 22.5 & 25.7 & 28.5 & 30.2 & 33.4 & 24.8 & 29.1 & 31.1 & 32.0 & 36.8 & 22.6 & 25.8 & 27.9 & 30.4 & 33.9 \\
\hline 5 & 21.5 & 3.7 & 26.7 & 31.4 & 36.3 & 24.5 & 27.5 & 32.3 & 34.4 & 37.3 & \begin{tabular}{|l|}
27.9 \\
\end{tabular} & 31.4 & 38.9 & 39.7 & \begin{tabular}{|l|}
43.2 \\
\end{tabular} & 24.6 & 27.5 & 32.6 & 35.2 & 38.9 \\
\hline 7 & 23.2 & 25.5 & 29.2 & 34.5 & 40.9 & 28.2 & 30.6 & 35.7 & 38.1 & 41.2 & 32.3 & 34.8 & 45.0 & 46.7 & 51.3 & 27.9 & 30.3 & 36.6 & 39.8 & 44.5 \\
\hline 9 & 24.5 & 27.9 & 32.5 & 36.4 & 44.5 & 30.3 & 33.4 & 38.2 & 44.5 & 48.1 & 30.5 & 39.3 & 47.5 & 49.6 & 53.4 & 28.4 & 33.5 & 39.4 & 43.5 & 48.7 \\
\hline $\bar{M}$ & 21.1 & 23.9 & 27.3 & 31.5 & 36.4 & 24.8 & 27.7 & 32.0 & 34.6 & 38.1 & 27.3 & 31.7 & 37.7 & 39.0 & 43.6 & & & & & \\
\hline
\end{tabular}

Table 3.9: Mean values of water loss (\%) of osmotically dehydrated bottle gourd at 60 Degree Celsius

\begin{tabular}{|c|c|c|c|c|c|c|c|c|c|c|c|c|c|c|c|c|c|c|c|c|}
\hline \multirow{4}{*}{$\mathbf{T}$} & \multicolumn{20}{|c|}{ Mass ratio } \\
\hline & \multicolumn{5}{|c|}{ 10:1 } & \multicolumn{5}{|c|}{$20: 1$} & \multicolumn{5}{|c|}{$\begin{array}{l}30: 1 \\
\end{array}$} & \multicolumn{5}{|c|}{ Mean } \\
\hline & \multicolumn{5}{|c|}{ Concentration (\%) } & \multicolumn{5}{|c|}{ Concentration (\%) } & \multicolumn{5}{|c|}{ Concentration (\%) } & \multicolumn{5}{|c|}{ Concentration (\%) } \\
\hline & 5 & 10 & 15 & 20 & 25 & 5 & 10 & 15 & 20 & 25 & 5 & 10 & 15 & 20 & 25 & 5 & 10 & 15 & 20 & 25 \\
\hline 1 & 20.6 & 21.5 & 25.2 & 26.5 & 20.1 & 21.6 & 22.5 & 25.4 & 27.7 & 31.2 & 24.6 & 25.9 & 26.4 & 28.8 & 32.6 & 22.3 & 23.3 & 25.7 & 27.7 & 28.0 \\
\hline 3 & 21.6 & 3.8 & 26.4 & 28.0 & 31.4 & 22.1 & 25.4 & 27.2 & 31.5 & 33.8 & 25.5 & 28.1 & 32.2 & 33.4 & 37.8 & 23.1 & 25.8 & 28.6 & 31.0 & 34.3 \\
\hline 5 & 22.4 & 24.6 & 28.8 & 31.4 & 36.3 & 25.2 & 28.3 & 31.5 & 35.3 & 37.5 & 31.2 & 34.2 & 39.2 & 41.4 & 46.0 & 26.3 & 29.0 & 33.2 & 36.0 & 39.9 \\
\hline 7 & 23.8 & 26.5 & 31.3 & 34.5 & 40.9 & 28.2 & 30.9 & 37.3 & 38.5 & 41.6 & 36.3 & 36.8 & 49.2 & 50.3 & 54.9 & 29.4 & 31.4 & 39.3 & \begin{tabular}{|l|}
41.1 \\
\end{tabular} & 45.8 \\
\hline 9 & 26.7 & 27.9 & 33.1 & 36.4 & 44.5 & 29.9 & 34.6 & 39.1 & 44.6 & \begin{tabular}{|l|l}
50.1 \\
\end{tabular} & 40.3 & 45.2 & 50.8 & 54.5 & 59.5 & 32.3 & 35.9 & 41.0 & 45.2 & 51.4 \\
\hline $\bar{M}$ & 23.0 & 24.9 & 29.0 & 31.4 & 34.6 & 25.4 & 28.3 & 32.1 & 35.5 & 38.8 & 31.6 & 34.0 & 39.6 & 41.7 & 46.2 & & & & & \\
\hline
\end{tabular}

\subsubsection{Weight reduction}

The results of weight reduction of bottle gourd cubes during the osmotic dehydration process for each after 1, 3, 5, 7 and 9 $h$, respectively. The mean values of weight reduction for the different set of experiments for presented in Table 3.10 through 3.12 .

The maximum weight reduction was $38.9 \%$ for samples osmotically dehydrate at $60^{\circ} \mathrm{C}$ for $9 \mathrm{~h}$ at $30: 1$ fruit to osmotic solution with $25 \%$ concentration. Whereas the minimum reduction in weight was found to be $10.7 \%$ for the samples osmotically dehydrated at $40^{\circ} \mathrm{C}$ for $1 \mathrm{~h}$ at $10: 1$ fruit to osmotic solution of $5 \%$ concentration. As can be observed that as the concentration of osmotic solution increased the weight reduction increased. This suggests that at same concentration of osmotic solution, higher temperature is responsible for increasing weight reduction. The same trends have been shown for three mass ratio 10:1, 20:1 and 30:1 (Table 3.10, 3.11 and 3.12.).

Table 3.10: Mean values of weight reduction (\%) of osmotically dehydrated bottle gourd at 40 Degree Celsius

\begin{tabular}{|c|c|c|c|c|c|c|c|c|c|c|c|c|c|c|c|c|c|c|c|c|}
\hline \multirow{4}{*}{$\mathbf{T}$} & \multicolumn{20}{|c|}{ Mass ratio } \\
\hline & \multicolumn{5}{|c|}{ 10:1 } & \multicolumn{5}{|c|}{ 20:1 } & \multicolumn{5}{|c|}{$\begin{array}{c}30: 1 \\
\end{array}$} & \multicolumn{5}{|c|}{ Mean } \\
\hline & \multicolumn{5}{|c|}{ Concentration (\%) } & \multicolumn{5}{|c|}{ Concentration (\%) } & \multicolumn{5}{|c|}{ Concentration (\%) } & \multicolumn{5}{|c|}{ Concentration (\%) } \\
\hline & 5 & 10 & 15 & 20 & 25 & 5 & 10 & 15 & 20 & 25 & 5 & 10 & 15 & 20 & 25 & 5 & 10 & 15 & 20 & 25 \\
\hline 1 & 10.7 & 10.7 & 12.2 & 12.8 & 16.5 & 13.6 & 15.8 & 16.2 & 17.7 & 18.9 & 13.5 & 15.7 & 16.1 & 16.8 & 18.8 & 12.6 & 14.1 & 14.8 & 15.8 & 18.1 \\
\hline 3 & 12.8 & 12.8 & 15.2 & 16.4 & 17.4 & 15.0 & 18.3 & 19.5 & 22.1 & 23.5 & 16.4 & 18.5 & 19.9 & 22.4 & 23.8 & 14.7 & 16.5 & 18.2 & 20.3 & 21.6 \\
\hline 5 & 12.3 & 12.3 & 15.1 & 16.0 & 17.0 & 14.6 & 17.5 & 18.7 & 21.6 & 23.0 & 15.8 & 17.6 & 19.6 & 21.5 & 22.4 & 14.2 & 15.8 & \begin{tabular}{|l|l}
17.8 \\
\end{tabular} & 19.7 & 20.8 \\
\hline 7 & 13.8 & 13.8 & 15.5 & 16.9 & 18.2 & 16.1 & 19.0 & 21.5 & 22.5 & 23.9 & 17.7 & 19.8 & 20.3 & 23.2 & 25.3 & 15.9 & 17.5 & \begin{tabular}{|l|}
19.1 \\
\end{tabular} & 20.9 & 22.5 \\
\hline 9 & 14.5 & 14.5 & 16.4 & 17.5 & 18.4 & 16.9 & 21.8 & 21.8 & 23.1 & 24.4 & 17.7 & 19.8 & 21.6 & 23.6 & 25.4 & 16.4 & 18.7 & \begin{tabular}{|l|}
19.9 \\
\end{tabular} & 21.4 & 22.7 \\
\hline $\mathrm{M}$ & 12.8 & 12.8 & 14.9 & 15.9 & 17.5 & 15.2 & 18.5 & 19.5 & 21.4 & 22.7 & 16.2 & 18.3 & 19.5 & 21.5 & 23.1 & & & & & \\
\hline
\end{tabular}


Table 3.11: Mean values of weight reduction (\%) of osmotically dehydrated bottle gourd at 50 Degree Celsius

\begin{tabular}{|c|c|c|c|c|c|c|c|c|c|c|c|c|c|c|c|c|c|c|c|c|}
\hline \multirow{4}{*}{$\mathbf{T}$} & \multicolumn{20}{|c|}{ Mass ratio } \\
\hline & \multicolumn{5}{|c|}{$10: 1$} & \multirow{2}{*}{\multicolumn{5}{|c|}{$\begin{array}{c}\text { 20:1 } \\
\text { Concentration }(\%)\end{array}$}} & \multirow{2}{*}{\multicolumn{5}{|c|}{$\begin{array}{c}\text { 30:1 } \\
\text { Concentration }(\%)\end{array}$}} & \multirow{2}{*}{\multicolumn{5}{|c|}{$\begin{array}{c}\text { Mean } \\
\text { Concentration (\%) }\end{array}$}} \\
\hline & \multicolumn{5}{|c|}{ Concentration (\%) } & & & & & & & & & & & & & & & \\
\hline & 5 & 10 & 15 & 20 & 25 & 5 & 10 & 15 & 20 & 25 & 5 & 10 & 15 & 20 & 25 & 5 & 10 & 15 & 20 & 25 \\
\hline 1 & 11.3 & 14.8 & 17.0 & 18.1 & 19.5 & 14.2 & 15.7 & 18.2 & 19.0 & 23.4 & 14.6 & 16.9 & 18.7 & 19.5 & 23.7 & 13.4 & 15.8 & 18.0 & 18.9 & 22.2 \\
\hline 3 & 15.4 & 17.0 & 18.3 & 19.2 & 19.8 & 17.6 & 20.8 & 22.4 & 23.3 & 24.8 & 18.5 & 20.6 & 22.5 & 23.8 & 25.5 & 17.2 & 19.5 & 21.1 & 22.1 & 23.4 \\
\hline 5 & 16.3 & 17.8 & 18.7 & 19.8 & 20.2 & 19.4 & 21.8 & 23.5 & 24.8 & 25.4 & 20.5 & 22.7 & 26.4 & 27.2 & 27.0 & 18.7 & 20.8 & 22.9 & 23.9 & 24.2 \\
\hline 7 & 17.9 & 19.0 & 19.5 & 20.5 & 20.3 & 22.6 & 24.5 & 26.1 & 26.6 & 26.6 & 23.8 & 26.1 & 31.8 & 32.3 & 29.8 & 21.4 & 23.2 & 25.8 & 26.5 & 25.6 \\
\hline 9 & 18.7 & 19.8 & 20.3 & 20.9 & 21.1 & 24.8 & 25.1 & 26.8 & 27.7 & 27.9 & 24.1 & 27.6 & 31.8 & 32.5 & 30.3 & 22.5 & 24.2 & 26.3 & 27.0 & 26.4 \\
\hline M & 15.9 & 17.7 & 18.8 & 19.7 & 20.2 & 19.7 & 21.6 & 23.4 & 24.3 & 25.6 & 20.3 & 22.8 & 26.2 & 27.1 & 27.3 & & & & & \\
\hline
\end{tabular}

Table 3.12: Mean values of weight reduction (\%) of osmotically dehydrated bottle gourd at 60 Degree Celsius

\begin{tabular}{|c|c|c|c|c|c|c|c|c|c|c|c|c|c|c|c|c|c|c|c|c|}
\hline \multirow{4}{*}{$\mathbf{T}$} & \multicolumn{20}{|c|}{ Mass ratio } \\
\hline & \multicolumn{5}{|c|}{ 10:1 } & \multicolumn{5}{|c|}{$\begin{array}{c}20: 1 \\
\end{array}$} & \multirow{2}{*}{\multicolumn{5}{|c|}{$\begin{array}{c}30: 1 \\
\end{array}$}} & \multicolumn{5}{|c|}{ Mean } \\
\hline & \multicolumn{5}{|c|}{ Concentration $(\%)$} & \multicolumn{5}{|c|}{ Concentration (\%) } & \multicolumn{2}{|c|}{ Concentration (\%) } & & & & \multicolumn{5}{|c|}{ Concentration (\%) } \\
\hline & 5 & 10 & 15 & 20 & 25 & 5 & 10 & 15 & 20 & 25 & 5 & 10 & 15 & 20 & 25 & 5 & 10 & 15 & 20 & 25 \\
\hline 1 & 15.1 & 16.3 & 18.2 & 20.6 & 22.5 & 15.5 & 16.4 & 18.9 & 20.6 & 22.9 & 17.9 & 18.6 & 18.1 & 19.6 & 22.5 & 16.2 & 17.1 & 18.4 & 20.3 & 22.6 \\
\hline 3 & 15.4 & 18.5 & 19.4 & 21.6 & 22.8 & 15.7 & 18.6 & 19.5 & 22.4 & 23.0 & 18.6 & 21.5 & 23.4 & 23.5 & 26.9 & 16.6 & 19.5 & 20.8 & 22.5 & 24.2 \\
\hline 5 & 16.9 & 19.4 & 20.8 & 22.4 & 23.1 & 16.8 & 19.2 & 20.7 & 27.8 & 23.1 & 22.5 & 23.3 & 27.8 & 28.2 & 30.9 & 18.7 & 20.6 & 23.1 & 26.1 & 25.7 \\
\hline 7 & 18.2 & 20.4 & 22.3 & 22.8 & 23.7 & 18.4 & 20.9 & 22.3 & 23.1 & 24.4 & 26.1 & 26.9 & 34.3 & 34.4 & 35.7 & 20.9 & 22.7 & 26.3 & 26.8 & 27.9 \\
\hline 9 & 19.4 & 22.8 & 23.4 & 27.0 & 28.9 & 19.6 & 22.4 & 23.6 & 27.9 & 29.2 & 28.2 & 31.1 & 34.5 & 36.2 & 38.9 & 22.4 & 25.4 & 27.2 & 30.4 & 32.3 \\
\hline $\mathrm{M}$ & 17.0 & 19.5 & 20.8 & 22.9 & 24.2 & 17.2 & 19.5 & 21.0 & 24.4 & 24.5 & 22.7 & 24.3 & 27.6 & 28.4 & 31.0 & & & & & \\
\hline
\end{tabular}

\subsection{Sensory evaluation}

Samples of osmotically dehydrated cubes prepared with the approximately central conditions of processing parameters $\left(50^{\circ} \mathrm{C}, 15 \%\right.$ concentration, $6 \mathrm{~h}$ and $20: 1$ mass ratio) were subjected to sensory evaluation to get an idea about the liking/disliking sample by the penalist. Sensory evaluation was performed in accordance with the nine-point hedonic scale. The average sensory score for different attributes, viz., appearance, colour, texture and overall acceptability for the bottle gourd cubes of size $1 \mathrm{~cm}$ are given are given in Table 4.13. it can be seen that average score obtained by each attribute is either 7 or above, this indicates that the sample was liked moderate or liked very much in respective attributes by the judges.

Table 4.13: Average score for different sensory attributes of osmotically dehydrated bottle gourd cubes.

\begin{tabular}{|c|c|c|c|}
\hline Appearance & Colour & Texture & Overall acceptability \\
\hline 7.5 & 7 & 7.7 & 7.6 \\
\hline
\end{tabular}

\section{Conclusion}

Based on the result, following conclusions were drawn

1. The maximum moisture content observed was $93.4 \%$ (wb) for samples osmotically dehydrated at $40^{\circ} \mathrm{C}$ for $1 \mathrm{~h}$ at 10:1 fruit to osmotic solution at $5 \%$ concentration. The minimum moisture content was $46.9 \%(\mathrm{wb})$ for samples osmotically dehydrated at $60^{\circ} \mathrm{C}$ for $9 \mathrm{~h}$ at $30: 1$ fruit to osmotic solution at $25 \%$ concentration.

2. As the duration incresesd, the weight reduction, water loss and solid gain of cubes were also increased for all the values of temperature and concentration. However, weight reduction, water loss and solid gain were very fast during the initial period of osmosis but equilibrium did reach even after $9 \mathrm{~h}$.

3. The solid gain varies from $21.4 \% \quad(9 \mathrm{~h}, 25 \%$ concentration, $60^{\circ} \mathrm{C}, 30: 1$ mass ratio) to from $4.7 \%$ (1h, $5 \%$ concentration, $40^{\circ} \mathrm{C}, 10: 1$ mass ratio).

4. The maximum water loss was $59.5 \%$ observed for samples osmotically dehydrated at $60^{\circ} \mathrm{C}$ for $9 \mathrm{~h}$ at $30: 1$ fruit to osmotic solution of $25 \%$ concentration. The minimum water loss was observed $14.5 \%$ for samples osmotically dehydrated at $40^{\circ} \mathrm{C}$ for $1 \mathrm{~h}$ at $10: 1$ fruit to osmotic solution of $5 \%$ concentration.

5. Weight reduction varies from $38.9 \%$ for samples osmotically dehydrated at $60^{\circ} \mathrm{C}$ for $9 \mathrm{~h}$ at $30: 1$ fruit to osmotic solution of $25 \%$ concentration to $10.7 \%$ for samples osmotically dehydrated at $40^{\circ} \mathrm{C}$ for $1 \mathrm{~h}$ at $10: 1$ fruit to osmotic solution of $5 \%$ concentration.

6. The sensory evaluation performed for the approximately central conditions processing parameters $\left(50^{\circ} \mathrm{C}, 15 \%\right.$ concentration, 6 h, 20:1 mass ratio) gave average scores of 7 or above.

The work contributes to the control of dehydration (WL) and impregnation (SG) process and given insights on the handling of solids and water profiles inside the products. Temperature was found to have a proportional effect on the water loss and the dry matter gain of osmotically dehydrates cubes. in order to satisfy the growing osmotic dehydration that produces intermediate moisture food will be increasingly used.

\section{References}

1. Alves SM, Silveira AM. Drying of tomatoes osmotically dehydrated and not osmotically dehydrated. Revista Universidade Rural Serie Ciencias Exatas e da Terra 2002; 21(Suplemento):21-30.

2. Bawa AS, Gujral HS. Effect of osmotic agents on the drying behaviour and product quality in raisin processing. Journal of Scientific and Induustrial Research. 2000; 59(1):63-66.

3. Bhgyalaxmi Hema P. Studies of osmotic dehydration of bottle gourd (Lagenaria siceraria). Submitted to Faculty of Agricultural Engineering, IGKVV, Raipur, (C.G.), 2006.

4. Giraldo Bedoya DP, Arango Velez LM, Marquez Cardozo CJ. Osmodehydration of blackberry (Rubus glaucus Benth) with three sweetening agents. Revista Facultad Nacional de Agronomia Medellin. 2004; 57(1):2253-2268.

5. Herwig T. Notes on Lagenaria and Cucurbita: Review and new contributions-Phyton (Horn, Austria). 2004; 44(2):245-308. 
6. Maeda M, Loreto RL. Osmotic dehydration of bananas. Semina Londrina. 1998; 19(1):60-67.

7. Nieto AB, Salvatori DM, Castro MA, Alzamora SM. Structural changes in apple tissue during glucose and sucrose osmotic dehydration. J Fd. Engg. 2004; 41(2):2431.

8. Rahman MS, Sablani SS, Al Ibrahim MA. Osmotic dehydration of potato: equilibrium kinetics. Drying Technology. 2001; 38(6):557-560.

9. Rahman MS, Lamb J. Osmotic dehydration of pineapple. Journal of Food science and Technology. 1990; 27(3):150-152.

10. Ranganna S. Hnadbook of analysis andquality control for fruit vegetables product, Tata Mcgraw Hill Publication Company Limited, New Delhi, 1995, 127-130.

11. Saputra D. Osmotic dehydration of pineapple. Drying Technology. 2001; 19(2):415-425.

12. Sereno AM, Mayor L, Moreira R. Dehydration of pumpkin using salt as osmotic agent: Evaluation of water and salt coefficients of diffusion. In: Drying Proceedings of the $14^{\text {th }}$ International Drying Symposium, Sao Paulo, Brazil, 2004, 22-258, 2157-2164.

13. Sunjka PS, Raghavan GSV. Assessment of pretreatment methods and osmotic dehydration for cranberries. Canadian Biosystems Engineering. 2004; 46:3.35-3.40.

14. Waliszewski Kubiak KN, Salgado Cervantes MA, Cortes Gonzalez HD, Romero Bello MI, Sanchez Solis AJ. Effect of $\mathrm{pH}$, temperature and sucrose concentration on its hydrolysis rate during osmotic dehydration process. Drying 94 Proceedings of $9^{\text {th }}$ International Drying Symposium, Gold Coast, Australia. 1994; 1-4(B):985991. 